\title{
Anomalous magnetic moments of free and bound leptons
}

\author{
Andrzej Czarnecki, Ulrich D. Jentschura, Krzysztof Pachucki, and \\ Vladimir A. Yerokhin
}

\begin{abstract}
We review the theoretical knowledge of anomalous magnetic moments of free electrons and muons, and of electrons bound in hydrogen-like ions. We discuss applications of those observables in the determination of fundamental physical constants, the fine structure constant and the electron mass, and in searches for new interactions.
\end{abstract}

PACS Nos.: 14.60.-z, 13.40.Em and 32.10.Dk

Résumé : French version of abstract (supplied by CJP if necessary)

[Traduit par la rédaction]

\section{Introduction}

A triumf of Dirac's relativistic theory was to explain the relationship between the electron's magnetic moment $\mu$ and its spin $s$. For any spin $1 / 2$ particle charge $q$ and mass $m$ one has

$\vec{\mu}=g \frac{q}{2 m} \vec{s}$,

where the proportionality coefficient $g$ is known as the Landé factor or a gyromagnetic ratio. For a pointlike particle, Dirac's theory predicts $g=2$.

In 1948 a deviation of $g$ from 2 was first observed [1]. An almost simultaneous calculation by Schwinger [2] showed how the electron self-interaction explains this deviation. Together with an observation and evaluation of a shift of the hydrogen spectrum due to a similar effect, those studies gave rise to Quantum Electrodynamics (QED). Detailed theoretical and experimental efforts extended those pioneering works, and we now know the values of $g-2$ with very high accuracy not only for a free electron but also for the muon, as well as for an electron bound in various atoms and ions.

In this talk we review the status of the theoretical knowledge of $g-2$ of free electrons and muons, and of electron bound in hydrogen-like ions such as the precisely studied five-fold ionized carbon. We also discuss how those quantities are used to determine fundamental physical constants and to search for new interactions.

Received September ??, 2005. Accepted ?????

Andrzej Czarnecki. Department of Physics, University of Alberta, Edmonton, AB T6G 2J1, Canada. e-mail: czar@phys.ualberta.ca

Ulrich D. Jentschura. Max-Planck-Institut für Kernphysik, Saupfercheckweg 1, 69117 Heidelberg, Germany. e-mail: Ulrich.Jentschura@mpi-hd.mpg.de

Krzysztof Pachucki. Institute of Theoretical Physics, Warsaw University, ul. Hoża 69, 00-681 Warsaw, Poland. e-mail: Krzysztof.Pachucki@ fuw.edu.pl

Vladimir A. Yerokhin. Department of Physics, St. Petersburg State University, Oulianovskaya 1, Petrodvorets, St. Petersburg 198504, Russia and Center for Advanced Studies, St. Petersburg State Polytechnical University, Polytekhnicheskaya 29, St. Petersburg 195251, Russia. e-mail: yerokhin@ pcqnt1.phys.spbu.ru 


\section{Free electron $g-2$ and the determination of $\alpha$}

The QED contribution to the electron $g-2$ is fundamental for the knowledge of the anomalous magnetic moments of all other leptons of interest. The differences which arise between the muon and the electron, or because of the binding of an electron in a light ion or atom, are small corrections to that dominant QED picture ${ }^{1}$. Thus it is crucial to know this part with the maximum possible accuracy.

Schwinger's calculation included a single virtual photon exchange - a one-loop contribution to the electron $g-2$. It was followed by studies of two- and three-loop effects which arrived at exact analytical results $[3,4,5,6,7]$. The four-loop contribution is known only numerically $[8,9]$.

Among contributions to $g-2$ there is a class universal for all charged leptons: those involving only virtual photons and closed charged lepton loops of the same fermion generation as the reference particle, but without particles of other flavors. Their characteristic feature is that the external lepton mass is their only mass scale. Thus they are given by numbers rather than functions of mass ratios and, since $g-2$ is dimensionless, contributions of analogous diagrams to the $g-2$ of other leptons are given by the same numbers. In addition, there are diagrams containing particles of different flavors they are functions of those additional masses and are different for each lepton.

For the electron, the first class dominates because diagrams containing other flavors are suppressed by powers of the small ratios of masses of the electron and the other particles.

It is convenient to introduce a notation for the deviation of $g$ from the Dirac value of 2 ,

$a \equiv \frac{g-2}{2}$

and we will use various sub- and super-scripts such as $a_{e}^{\mathrm{QED}}$ to denote contributions to various leptonic magnetic anomalies from various physical effects. Thus, the theoretical prediction for the QED contribution to the electron $g-2$ is given by a (truncated) series in the fine structure constant,

$a_{e}^{\mathrm{QED}}=\sum_{n=1}^{4} A_{n}\left(\frac{\alpha}{\pi}\right)^{n}+\left[B_{2}(e, \mu)+B_{2}(e, \tau)\right]\left(\frac{\alpha}{\pi}\right)^{2}+B_{3}(e, \mu)\left(\frac{\alpha}{\pi}\right)^{3}+\mathcal{O}\left(\alpha^{5}, m_{e}^{2} \alpha^{4} / m_{\mu}^{2}\right)$.

Here $A_{n}$ are the universal, "pure QED” effects, and $B_{n}\left(l, l^{\prime}\right)$ describe how the loops containing lepton $l^{\prime}$ affect $a_{l}$. The universal contributions are $[2,3,5,7,9]$ :

$$
\begin{aligned}
A_{1}= & \frac{1}{2} \\
A_{2}= & \frac{3}{4} \zeta_{3}-\frac{\pi^{2}}{2} \ln 2+\frac{\pi^{2}}{12}+\frac{197}{144} \\
\approx & -0.3284789656 \\
A_{3}= & \frac{83}{72} \pi^{2} \zeta_{3}-\frac{215}{24} \zeta_{5}-\frac{239}{2160} \pi^{4}+\frac{139}{18} \zeta_{3}+\frac{25}{18}\left[24 \operatorname{Li}_{4}\left(\frac{1}{2}\right)+\ln ^{4} 2-\pi^{2} \ln ^{2} 2\right] \\
& -\frac{298}{9} \pi^{2} \ln 2+\frac{17101}{810} \pi^{2}+\frac{28259}{5184} \\
\approx & 1.1812415 \\
A_{4}= & -1.7283(35) .
\end{aligned}
$$

The non-universal coefficients $B$ can be found, for example, in $[10,11]$. Their effect is dominated by the two-loop diagram containing a muon loop. 1 However, as we will see later, the binding corrections grow like $Z^{2}$ and for a hydrogen-like carbon ion are about half the size
of the QED effect. Fortunately this leading part of binding effects is known to all orders in $Z \alpha$, see Eq. (9). 
In addition, $a_{e}$ is slightly affected by the electron interactions with virtual weak bosons $W$ and $Z$, and by hadronic loop insertions in the photon propagator. Those effects are much more important for the muon and will be discussed below. For the electron, they increase $a_{e}$ by $1.7 \times 10^{-12}$ [9].

The prediction for $a_{e}$ is dominated by the coefficients $A$ given in eq. (4). We see that it is given by a sign-alternating and thus very well convergent series in the small parameter $\alpha / \pi$. We can compare this prediction with the measurement results [12],

$a_{e^{-}}^{\exp }=1159652188.4(4.3) \times 10^{-12}$,

$a_{e^{+}}^{\exp }=1159652187.9(4.3) \times 10^{-12}$,

to get the best present value of the fine structure constant,

$\alpha=1 / 137.03599883(51)$.

The error is at present dominated by the experimental uncertainty. However, the theoretical errors due to $A_{4}$ and even more important due to the unknown five-loop effects, are on the order of $1 / 15$ of the present experimental error. A new measurement is expected soon [13] and is planned to reach just that precision level. It is very important to improve the theoretical prediction.

\section{Bound electron $g$ and the determination of $m_{e}$}

Here we discuss how the $g$ factor of an electron is modified not only by the electron self-interaction but also by its interactions with an external Coulomb field, for example in a simple atom. We also show how one uses this knowledge to obtain the best determination of the electron mass.

The electron mass $m_{e}$ is a fundamental physical constant which sets the scale of all atomic energy levels. Its accurate value, together with the fine structure constant $\alpha$, are necessary for precise predictions of atomic transition spectra, line widths and transition rates. $m_{e}$ expressed in terms of the atomic mass unit $u$, equal to the $1 / 12$ of the ${ }^{12} \mathrm{C}$ atom mass, is also sensitive to the possible time variation of the strength of fundamental interactions, since $m_{e}$ is, as is presently believed, determined by the electron Yukawa coupling to the Higgs field, while the atomic mass is primarily due to strong interactions. It is of great value to improve our knowledge of $m_{e}$ as far as allowed by the present experimental capabilities and theoretical knowledge.

Until recently, the best determination of $m_{e}$ was possible by measuring the cyclotron frequency $\nu_{c}$ (free) of a single electron observed in a Penning trap,

$\nu_{c}($ free $)=-\frac{e B}{m_{e}}$

Here $B$ is the magnetic field acting on the electron and $e$ is the physical charge of the electron, $e<0$. Since the $B$ field in general is relatively poorly known, one usually measures the cyclotron precession $\nu_{c}$ of another particle such as a carbon ion in the same field and determines the ratio of masses of the electron and that particle. The disadvantage of this method is that it is difficult to maintain the same strength of the $B$ field for the measurements of both frequencies. The best electron mass value obtained with this method is accurate to 2.2 parts per billion (ppb) [14].

It has recently been discovered that one can improve the knowledge of $m_{e}$ using hydrogen-like ions. A five-fold ionized ${ }^{12} \mathrm{C}$ atom in the Penning trap allows one to study the Larmor precession frequency of the electron $\nu_{L}$ and the cyclotron frequency of the ion $\nu_{c}\left({ }^{12} \mathrm{C}^{5+}\right)$. The electron and ion mass ratio (and thus $m_{e} / u$ ) is found by taking the ratio of both frequencies,

$\frac{m_{e}}{m\left({ }^{12} \mathrm{C}^{5+}\right)}=\frac{g_{\text {bound }}}{10} \frac{\nu_{c}\left({ }^{12} \mathrm{C}^{5+}\right)}{\nu_{\mathrm{L}}}$. 
Since both frequencies are measured simultaneously, there is no difficulty in maintaining the magnetic field strength. Thus, a recent measurement $[15,16]$ (see also [17]) has reached the experimental precision of one half part per billion, $0.5 \mathrm{ppb}$ - four times better than that of the free electron determination. However, the gyromagnetic factor $g_{\text {bound }}$ of the electron bound in the ion is modified by the binding effects and is not nearly as well known as that of the free electron.

Although the theoretical interest in $g_{\text {bound }}$ has greatly increased only recently in connection with the determination of $m_{e}$, its first study goes back two decades before Schwinger's calculation of the one-loop QED effect. In 1928, in one of the first calculations using the Dirac equation, Breit [18] determined the magnetic moment of the electron in a hydrogen-like atom with the atomic number $Z$ and found

$g_{\text {bound }}($ Dirac eq. $)=\frac{2}{3}\left(1+2 \sqrt{1-(Z \alpha)^{2}}\right)$,

a result notable for being exact to all orders in binding effects. In the limit $Z \alpha \rightarrow 0$ we get $g=2$, the prediction of the Dirac theory.

With the onset of QED, it became clear that the so-called loop effects, due to the self-interaction of the electron, free or bound, modify its $g$ factor, as we discussed in Section 2. The bound electron $g$ factor can be described as a series in $\alpha / \pi$, but in contrast to the free electron case the coefficients are now functions of the binding effects parametrized by $Z \alpha$,

$\Delta g(\mathrm{QED})=f_{1}(Z \alpha) \frac{\alpha}{\pi}+f_{2}(Z \alpha)\left(\frac{\alpha}{\pi}\right)^{2}+f_{3}(Z \alpha)\left(\frac{\alpha}{\pi}\right)^{3}+\ldots$

The values of the coefficient functions $f_{1}, f_{2}, \ldots$ at $Z \alpha \rightarrow 0$ describe the anomalous magnetic moment of a free electron. As we have already seen, the first three of them are known exactly and the fourth one numerically. The main challenge in the theory of $g_{\text {bound }}$ is to determine the dependence of $f_{1}$ and $f_{2}$ on $Z \alpha$ (for the presently planned experiments the free values of further coefficients are sufficient).

The linear and cubic terms in the expansion of the coefficient functions vanishes and the quadratic term is universal [19]. The coefficient functions differ only in the quartic term,

$f_{j}(Z \alpha)=2 A_{j}\left[1+\frac{(Z \alpha)^{2}}{6 n^{2}}\right]+(Z \alpha)^{4}\left(h_{41}^{(j)} \ln \frac{1}{Z \alpha}+h_{40}^{(j)}\right)+\mathcal{O}\left((Z \alpha)^{5}\right)$.

In writing down this equation we have assumed that the electron is in the $n S$ state. The known coefficients $A_{j}$ are listed in Eq. (4). The function $f_{1}$ has been determined numerically to all orders in $Z \alpha$ $[20,21,22,23,24]$. However, because of the limited numerical accuracy, it was essential to analytically determine the fourth-order term in the $Z \alpha$ expansion, valid for low- $Z$ systems such as the carbon ion $(Z=6)$. Such a study was performed very recently [25].

For the function $f_{2}$, a numerical study has not been performed. In $[25,26]$, the coefficient $h_{41}^{(2)}$ of the logarithmic term and the value of $h_{40}^{(2)}$ were found. These result shift the central value of $m_{e}$ and remove the main source of its theoretical uncertainty.

The only known way of analytically determining high-order effects such as those contributing to $h_{40}^{(2)}$ is to study the various photon energy regions separately, using a non-relativistic effective theory based on QED, known as NRQED [27]. However, that separation of scales is plagued by technical difficulties, some of which have been solved only recently.

The hard-photon effects are evaluated neglecting the binding of the electron in an atom, using the scattering amplitude of a free electron in external magnetic and electric fields. That scattering amplitude determines a potential of the form $Z \alpha \vec{\sigma} \cdot \vec{B} \delta^{d}(r)$ ( $\sigma_{i}$ are the Pauli matrices) whose expectation value with the Schrödinger electron wave function gives the correction to the electron $g$ factor.

There are two difficulties related to the hard-scale region. First, taken separately, its contribution is divergent. This is because the electron binding effects that we neglect become important when photons 
$\gamma$ have wavelengths similar to the atomic dimension (an infrared singularity). Second, there is a danger of counting twice some contributions of iterated effects of order $\alpha / \pi$.

The first problem is best solved by applying to this atomic physics problem a tool developed in high-energy physics: dimensional regularization (DR). The DR has been instrumental in studies of positronium [28, 29] and helium [30]. We deal with the second, double-counting problem, by subtracting from corrections to a single external field coupling a contribution of electric form factors. The form factors must of course be calculated to the appropriate order in the coupling constant and external field momentum.

As a result we obtained $[25,26]$ the formula for an electron bound in a hydrogen-like atom in any $n S$ state,

$$
\begin{aligned}
g(n S)= & g^{(0)}+g^{(1)}+g^{(2)}+\mathcal{O}\left(\alpha^{3}\right) \\
g^{(0)}= & 2-\frac{2}{3} \frac{(Z \alpha)^{2}}{n^{2}}+\left(\frac{1}{2 n}-\frac{2}{3}\right) \frac{(Z \alpha)^{4}}{n^{3}}, \\
g^{(1)}= & \frac{\alpha}{\pi}\left\{1+\frac{(Z \alpha)^{2}}{6 n^{2}}+\frac{(Z \alpha)^{4}}{n^{3}}\left[\frac{32}{9} \ln \left[(Z \alpha)^{-2}\right]+\frac{73}{54}-\frac{5}{24 n}-\frac{8}{9} \ln k_{0}-\frac{8}{3} \ln k_{3}\right]\right\}, \\
g^{(2)=} & \left(\frac{\alpha}{\pi}\right)^{2}\left\{2 A_{2}\left(1+\frac{(Z \alpha)^{2}}{6 n^{2}}\right)\right. \\
& +\frac{(Z \alpha)^{4}}{n^{3}}\left[\frac{28}{9} \ln \left[(Z \alpha)^{-2}\right]+\frac{258917}{19440}-\frac{4}{9} \ln k_{0}-\frac{8}{3} \ln k_{3}+\frac{113}{810} \pi^{2}\right. \\
& \left.\left.-\frac{379}{90} \pi^{2} \ln 2+\frac{379}{60} \zeta(3)+\frac{1}{n}\left(-\frac{985}{1728}-\frac{5}{144} \pi^{2}+\frac{5}{24} \pi^{2} \ln 2-\frac{5}{16} \zeta(3)\right)\right]\right\} .
\end{aligned}
$$

In all the above results we have neglected terms of order $(Z \alpha)^{5}$ and higher. The numerical values of $\ln k_{0}$ and $\ln k_{3}$ for the lowest $(n=1) S$ state are

$\ln k_{0}(1 S)=2.984128556$,
$\ln k_{3}(1 S)=3.272806545$.

Their values for several higher $S$ states are given in [26].

In order to use our QED result to obtain the electron mass we included also small numerical corrections arising from the finite nuclear size and mass, higher powers of $(Z \alpha)$, etc. (for a detailed discussion and references see [26]). Using the results of measurements with carbon [15] and oxygen $[16]$ we found $[25,26]$ the following values for the electron mass (in atomic mass units):

$$
\begin{aligned}
& m\left({ }^{12} \mathrm{C}^{5+}\right)=0.00054857990932(29), \\
& m\left({ }^{16} \mathrm{O}^{7+}\right)=0.00054857990960(41) .
\end{aligned}
$$

The uncertainty of these results originates from the experimental value for the ratio of the electronic Larmor precession frequency and the cyclotron frequency of the ion in the trap; the uncertainty due to the theoretical prediction is smaller by more than an order of magnitude.

\section{Free muon $g-2$ and the search for new physics}

Muon has the same interactions as the electron but since it is much heavier, $m_{\mu} \simeq 207 m_{e}$, heavy particles such as hadrons and the electroweak bosons affect its properties much stronger. For the same reason, $a_{\mu} \equiv\left(g_{\mu}-2\right) / 2$ is a much more sensitive probe of exotic phenomena ("new physics") than $a_{e}$. Indeed, we shall see that there is a tantalizing disagreement between the Standard Model prediction and the experiment that may be due to an intervention of yet unknown phenomena such as supersymmetry. 
Most of the muon magnetic anomaly $a_{\mu}$ is due to QED. All but sixty parts per million in $a_{\mu}$ arise from pure QED contributions, that is diagrams with photons and leptons only. This poses a problem from the point of view of searching for more exotic phenomena, because the QED prediction must reach a very high accuracy.

The QED result can be represented in a perturbative series in the fine structure constant,

$a_{\mu}^{\mathrm{QED}}=\sum C_{n}\left(\frac{\alpha}{\pi}\right)^{n}$

As discussed in Section 2, there is a universal part of the QED contributions (included in $C_{n}$ ), common to the electron and the muon. For the electron $g-2$, that universal part dominated. The physics of the muon $g-2$ is quite different beginning with the two-loop contributions, where the non-univeral parts first appear as vacuum polarization insertions in the photon propagator. The higher-order QED contributions to $a_{\mu}$ are dominated by diagrams containing electron loops, and are significantly enhanced compared to the electron case.

The source of this enhancement are logarithms of the ratio of the muon to electron mass squared, $\ln \frac{m_{\mu}^{2}}{m_{e}^{2}} \simeq 11$. In the diagrams studied so far, the electron loop effects are either vacuum polarization insertions in the photon propagator or so-called light-by-light diagrams, with four photons attached to the electron loop. Typically, we find one power of the large logarithm for each electron loop, with the light-by-light diagrams additionally enhanced by factors of $\pi^{2}$. Having identified those leading contributions it is possible, although still very challenging, to estimate their value at the five-loop level and thus obtain an approximation for $C_{5}[10,31]$.

The resulting QED contribution to $a_{\mu}$ becomes [10]

$a_{\mu}^{\mathrm{QED}}=116584717.88(89) \times 10^{-11}$.

Another difference between the electron and the muon $g-2$ is that for the latter hadronic loops are of considerable importance. The hadronic contribution to $a_{\mu}$ is about twenty thousand times smaller than the QED part, but it is responsible for almost all of the Standard Model uncertainty in $a_{\mu}$ (about a hundred times more than the QED).

The hadronic contributions can be divided up into three parts: the leading order vacuum polarization, higher-order QED corrections to it, and the light-by-light part,

$a_{\mu}^{\mathrm{had}}=a_{\mu}^{\mathrm{had}}(\mathrm{LO})+a_{\mu}^{\mathrm{had}}(\mathrm{HO})+a_{\mu}^{\mathrm{had}}(\mathrm{LBL})$.

The hadronic vacuum polarization contribution can be determined from $e^{+} e^{-}$annihilation into hadrons or from hadronic $\tau$-lepton decays. The connection with the muon $g-2$ is more direct in the case of the $e^{+} e^{-}$annihilation data, but the $\tau$ hadronic decays give better statistical information in the low energy region, where studies of the $e^{+} e^{-}$annihilation are very difficult. Unfortunately, the theoretical input needed for the translation of the hadronic $\tau$ decay spectrum into the muon $g-2$ contribution is not fully understood, as has become clear over the last couple of years. As a result, the present consensus is that the $\tau$ data cannot be reliably used before further theoretical progress is made [32].

Fortunately, the accuracy of the $e^{+} e^{-}$data has recently improved, thanks largely to new results from Novosibirsk [33]. In addition, a new source of information on the low-energy annihilation has been discovered in radiative return processes [34]. For the purpose of the present discussion we adopt the result given in [35],

$a_{\mu}^{\text {had }}(\mathrm{LO})=6934(64) \times 10^{-11}$.

The higher-order QED corrections to this result have recently been reevaluated [36]. Their updated value is

$a_{\mu}^{\text {had }}(\mathrm{HO})=-98(1) \times 10^{-11}$. 
An important recent development is the analysis of the light-by-light diagrams by Melnikov and Vainshtein [37]. In the past, similar calculations were done using models of low-energy hadronic interactions with electromagnetic currents. This involved assumptions about interactions of photons with light hadrons, especially pions. The new study has shown that some of those assumptions were misleading. The crucial tool is the operator product expansion and the determination of operator coefficients using perturbative QCD calculations. One important finding concerns the interaction $\pi^{*} \gamma^{*} \gamma$ in the limit of the soft real photon. In contrast to the previously assumed "vector meson dominance" picture, in which the virtual photon's interaction is described by a formfactor (involving an additional factor $1 /\left(Q^{2}+m_{V}^{2}\right)$, with $m_{V}$ on the order of the $\rho$ meson mass), it is now found that in the soft real photon limit there is no such formfactor present. This enhances the large-virtuality contributions at $Q^{2} \gtrsim m_{\rho}^{2}$ and thus enhances the total light-by-light contribution.

More efforts in improving the accuracy of this part of the prediciton are clearly needed. In particular, it is important to better determine or constrain effects sub-leading in the $1 / N_{c}$ expansion, found to be consistent with zero within the accuracy aimed for in [37]. Since some model calculation find negative contributions of such effects, here we take a slightly smaller total value of $a_{\mu}^{\mathrm{LBL}}(\mathrm{had})$ than in [37] and increase its error,

$a_{\mu}^{\text {had }}(\mathrm{LBL})=120(40) \times 10^{-11}$.

Taking together the three parts of the hadronic effect, Eqs. $(18,19,20)$, we find

$a_{\mu}^{\text {had }}=6956(75) \times 10^{-11}$.

The last source of the Standard Model corrections is the electroweak sector. Similarly to the QED part, it is perturbatively calculable, with the small uncertainties arising from the unknown Higgs boson mass and from the small hadronic contributions, primarily to the induced coupling between the $Z$ boson and two photons. The electroweak effects arise at one loop: there is a $Z$ boson loop, similar to the lowest-order QED diagram and a $W$ contribution with the muon neutrino. Relative to the QED contribution, they are suppressed by the factor $m_{\mu}^{2} / M_{W}^{2}$,

$a_{\mu}^{\mathrm{EW}} \sim \frac{m_{\mu}^{2}}{M_{W}^{2}} a_{\mu}^{\mathrm{QED}} \simeq 200 \times 10^{-11}$.

There is also a one-loop diagram with the Higgs boson, but it contains two extra factors of $m_{\mu} / M_{W} \simeq$ $10^{-3}$ and is negligible. The Higgs effect is in fact much larger at the two-loop level, because those two tiny factor are replaced by just one $\frac{\alpha}{\pi}$ : at two loops the Higgs can couple once to the muon, giving the chirality flip needed for the anomalous magnetic moment type of interaction, and once to some virtual heavy particle. Thus, such diagrams have no light-mass suppression relative to other two-loop diagrams.

Some of the two-loop electroweak contributions are enhanced by logarithms of the muon-to- $W$ mass ratio [38]. The two-loop calculation, including complete logarithmic and non-logarithmic terms was carried out in [39, 40], and was recently confirmed [41, 42]. Even the leading-logarithmic threeloop result is now known $[43,44]$. Together, one finds

$a_{\mu}^{\mathrm{EW}}=154(2.2) \times 10^{-11}$.

Using Eqs. $(17,21,23)$ we can now put together the full Standard Model prediction for the muon $g-2$,

$a_{\mu}^{\mathrm{SM}}=a_{\mu}^{\mathrm{QED}}+a_{\mu}^{\mathrm{had}}+a_{\mu}^{\mathrm{EW}}=116591828(75) \times 10^{-11}$.

How does this number compare with the experimental results? We now know the final results of the Brookhaven measurments for both negative and positive muons. For the negative muon $a_{\mu}$ one has [45],

$a\left(\mu^{-}\right)=116592140(80)(30) \times 10^{-11}$, 
slightly higher than the earlier measurement for the positive muon [46],

$a\left(\mu^{+}\right)=116592040(70)(50) \times 10^{-11}$.

However, the two results agree within their experimental errors. When all the measurements for positive and negative muons are averaged, one arrives at the world average $a_{\mu}$ [45],

$a_{\mu}(\exp )=116592080(60) \times 10^{-11}$,

where the relative error is only one half of a part per million.

We see that the theoretical prediction Eq. (24) falls short of the experimental world-average, Eq. (27), by $252(96) \times 10^{-11}$, or 2.6 standard deviation. With all the scrutiny that has been given to all parts of the theoretical prediction and experimental inputs, it is difficult to envision that the Standard Model can explain this difference. It is, however, still very important to make efforts to improve the theoretical accuracy: the most imporant tasks seem to be an improvement of the hadronic LBL part and an independent study of four- and five-loop QED effects. Extremely important would be a determination of the isospin-breaking corrections, enabling us to use hadronic $\tau$ decays for the hadronic VP part.

\section{Summary}

Anomalous magnetic moments are of great current experimental and theoretical interest. Very precise measurements of the positive and negative muon $g-2$ have recently been completed in the Brookhaven National Laboratory. A new measurement of the free electron $g-2$ is under preparation at Harvard University. Further measurements are also planned for the electron bound in hydrogen-like ions (such as calcium) and in ions with a few electrons.

It is thus essential to continue theoretical efforts to match the improving measurements. For the free electron, an independent evalulation of four-loop effects is crucial. It is equally important to estimate in a reliable way the five-loop effect, perhaps by identifying the diagrams responsible for the largest contributions. This requires a new theoretical insight. For the bound electron, a non-perturbative numerical study of the two-loop effects for all values of the nuclear charge $Z$ would be most valuable, as well as its matching with the known analytical terms for low $Z$. It is also very interesting to further develop methods to determine $g$ of systems with several electrons. For the free muon, the biggest challenge is to improve the knowledge of hadronic effects, both vacuum polarization and light-by-light. Perhaps lattice calculations will help, but further studies of low-energy interactions are also very important.

Anomalous magnetic moments of leptons certainly remain a fascinating field of research, with many expected experimental improvements and open theoretical questions.

\section{Acknowledgments}

This work was supported by EU grant No. HPRICT- 2001-50034 and by RFBR grant No. 04-0217574. A.C. acknowledges support by the Science and Engineering Research Canada. V.A.Y. acknowledges support by the foundation Dynasty. U.D.J. acknowledges support from the Deutsche Forschungsgemeinschaft via the Heisenberg program.

\section{References}

1. P. Kusch and H. M. Foley, Phys. Rev. 74, 250 (1948).

2. J. Schwinger, Phys. Rev. 73, 416 (1948).

3. C. M. Sommerfield, Phys. Rev. 107, 328 (1957).

4. C. M. Sommerfield, Ann. Phys. 5, 26 (1958).

5. A. Petermann, Nucl. Phys. 3, 689 (1957).

6. R. Z. Roskies, M. J. Levine, and E. Remiddi, in Quantum Electrodynamics, edited by T. Kinoshita (World Scientific, Singapore, 1990), pp. 162-217. 
7. S. Laporta and E. Remiddi, Phys. Lett. B379, 283 (1996).

8. T. Kinoshita and M. Nio, Phys. Rev. Lett. 90, 021803 (2003).

9. T. Kinoshita and M. Nio, hep-ph/0507249 (unpublished).

10. T. Kinoshita, Nucl. Phys. Proc. Suppl. 144, 206 (2005).

11. A. Czarnecki and W. J. Marciano, Nucl. Phys. B (Proc. Suppl.) 76, 245 (1999).

12. R. S. van Dyck Jr., P. B. Schwinberg, and H. G. Dehmelt, Phys. Rev. Lett. 59, 26 (1987).

13. G. Gabrielse, talk at the International Symposium Lepton Moments, Cape Cod, 2003 (unpublished).

14. D. L. Farnham, R. S. Van Dyck, Jr., and P. B. Schwinberg, Phys. Rev. Lett. 75, 3598 (1995).

15. H. Häffner et al., Phys. Rev. Lett. 85, 5308 (2000).

16. J. Verdú et al., Phys. Rev. Lett. 92, 093002 (2004).

17. T. Beier et al., Phys. Rev. Lett. 88, 011603 (2002).

18. G. Breit, Nature 122, 649 (1928).

19. H. Grotch, Phys. Rev. Lett. 24, 39 (1970).

20. A. Blundell, K. T. Cheng, and J. Sapirstein, Phys. Rev. A55, 1857 (1997).

21. H. Persson, S. Salomonson, P. Sunnergren, and I. Lindgren, Phys. Rev. A56, R2499 (1997).

22. T. Beier et al., Phys. Rev. A62, 032510 (2000).

23. V. A. Yerokhin, P. Indelicato, and V. M. Shabaev, Phys. Rev. Lett. 89, 143001 (2002).

24. V. A. Yerokhin, P. Indelicato, and V. M. Shabaev, Phys. Rev. A69, 052503 (2004).

25. K. Pachucki, U. D. Jentschura, and V. A. Yerokhin, Phys. Rev. Lett. 93, 150401 (2004), erratum: Phys. Rev. Lett. 94, 229902 (2005).

26. K. Pachucki, A. Czarnecki, U. D. Jentschura, and V. A. Yerokhin, Phys. Rev. A72, 022108 (2005).

27. W. E. Caswell and G. P. Lepage, Phys. Lett. B167, 437 (1986).

28. A. Pineda and J. Soto, Phys. Rev. D59, 016005 (1999).

29. A. Czarnecki, K. Melnikov, and A. Yelkhovsky, Phys. Rev. Lett. 82, 311 (1999).

30. V. Korobov and A. Yelkhovsky, Phys. Rev. Lett. 87, 193003 (2001).

31. A. L. Kataev, hep-ph/0507174 (unpublished).

32. M. Davier and W. J. Marciano, Ann. Rev. Nucl. Part. Sci. 54, 115 (2004).

33. S. I. Eidelman, Nucl. Phys. Proc. Suppl. 144, 223 (2005).

34. J. H. Kuhn, Nucl. Phys. Proc. Suppl. 144, 214 (2005).

35. M. Davier, Nucl. Phys. Proc. Suppl. 144, 250 (2005).

36. K. Hagiwara, A. D. Martin, D. Nomura, and T. Teubner, Phys. Rev. D69, 093003 (2004).

37. K. Melnikov and A. Vainshtein, Phys. Rev. D70, 113006 (2004).

38. T. V. Kukhto, E. A. Kuraev, A. Schiller, and Z. K. Silagadze, Nucl. Phys. B371, 567 (1992).

39. A. Czarnecki, B. Krause, and W. Marciano, Phys. Rev. D52, 2619 (1995).

40. A. Czarnecki, B. Krause, and W. Marciano, Phys. Rev. Lett. 76, 3267 (1996).

41. S. Heinemeyer, D. Stockinger, and G. Weiglein, Nucl. Phys. B690, 62 (2004).

42. T. Gribouk and A. Czarnecki, University of Alberta preprint (unpublished).

43. A. Czarnecki, W. J. Marciano, and A. Vainshtein, Phys. Rev. D67, 073006 (2003).

44. G. Degrassi and G. F. Giudice, Phys. Rev. D58, 053007 (1998).

45. G. W. Bennett et al., Phys. Rev. Lett. 92, 161802 (2004).

46. G. W. Bennett et al., Phys. Rev. Lett. 89, 101804 (2002). 